Relations industrielles

Industrial Relations

\title{
La nouvelle loi de la Fonction Publique du Québec
}

\section{Jean-Réal Cardin}

Volume 21, numéro 2, 1966

URI : https://id.erudit.org/iderudit/027678ar

DOI : https://doi.org/10.7202/027678ar

Aller au sommaire du numéro

Éditeur(s)

Département des relations industrielles de l'Université Laval

ISSN

0034-379X (imprimé)

1703-8138 (numérique)

Découvrir la revue

Citer cet article

Cardin, J.-R. (1966). La nouvelle loi de la Fonction Publique du Québec.

Relations industrielles / Industrial Relations, 21(2), 251-257.

https://doi.org/10.7202/027678ar

Tous droits réservés @ C Département des relations industrielles de l'Universite Laval, 1966
Ce document est protégé par la loi sur le droit d'auteur. L’utilisation des services d'Érudit (y compris la reproduction) est assujettie à sa politique d'utilisation que vous pouvez consulter en ligne.

https://apropos.erudit.org/fr/usagers/politique-dutilisation/ 


\section{COMMENTAIRES}

\section{LA NOUVELLE LOI DE LA FONCTION PUBLIQUE DU QUÉBEC}

\section{Jean-Réal Cardin}

LE RÉGIME ANTÉRIEUR

La distinction traditionnelle entre droit public et droit privé n'est plus aussi étanche qu'elle l'était. Avec l'intervention accélérée de l'Etat dans l'économie, nous assistons à ce que Jean Lacroix, à la suite des juristes français, appelle la privatisation du public, et la publicisation du privé. C'est là, en somme, du point de vue des relations industrielles, toute l'histoire du droit moderne du travail. En stricte analyse juridique, à venir jusqu'à présent, la fonction publique ne ressortissait pas au droit du travail; elle s'inscrivait plutôt dans les schèmes du droit administratif, lui-même une partie du droit public. Il s'agissait donc d'un " statut " et non d'un " contrat " conclu entre personnes morales, autonomes et également responsables devant la loi, comme c'est le cas en droit privé. Nous avions donc affaire à un "service " civil, comme nous avons le service pour fins militaires ou de police.

Dans une telle analyse, il ne pouvait être question d'un « contrat * de travail entre l'Etat souverain et son « serviteur ». Les conditions de travail de ce dernier lui étaient "statutairement ", unilatéralement imposées par l'Etat, ainsi que tout le régime des sanctions en cas d'infraction de sa part dans l'exercice de ses fonctions. Pas de négociation possible donc dans un tel régime, et encore moins de convention collective et de grève, qui sont autant de notions battant en brèche celle même du caractère souverain de l'Etat.

Cependant, si l'on reconnaît que l'Etat devient le plus gros employeur des temps modernes, il faut reconnaître aussi que de plus en plus, le droit du travail d'origine privée doit tendre à régir les rapports qu'il entretient avec ses employés. Tout comme, d'autre part, des éléments de plus en plus nombreux du droit administratif, donc public, conditionnent les relations employeurs-employés dans le secteur privé de l'économie.

Si nous nous rendons à cette évidence, nous ne pouvons plus, au nom de la souveraineté de l'Etat-gouvernement, lui appliquer, en tant qu'Etat-employeur, la pure logique du droit administratif et refuser à ses employés l'essentiel des libertés garanties aux autres salariés par le droit du travail. 
En conséquence, si le droit du travail doit servir davantage à analyser les relations entre l'Etat-employeur et la fonction publique, il $s$ 'ensuit que l'organisation et la sécurité syndicales, la négociation et la convention collectives, ainsi que des modes adaptés de règlement des conflits individuels et collectifs, doivent être octroyés aux employés de la fonction publique. Qu'en a-t-il été jusqu'ici, c'est-à-dire, jusqu'au ler septembre 1964, date d'entrée en vigueur du présent Code du Travail? Sommairement la situation était la suivante: rien dans notre loi des Syndicats professionnels, ni dans celles des Relations ouvrières ni des Différends entre les Services publics et leurs salariés, n'excluait les fonctionnaires gouvernementaux de leur application; au contraire, dans cette dernière loi, les fonctionnaires étaient nommément inclus dans la nomenclature des services publics. Il pouvait donc sembler que l'ensemble des droits reconnus par ces textes s'appliquaient à ces derniers comme aux autres salariés couverts par eux. En réalité la situation était tout autre à notre avis. Au sous-paragraphe $5 \mathrm{du}$ paragraphe « $\mathrm{d}$ » de l'article 2 de la loi des Différends entre les Services publics et leurs salariés, le législateur avait bien pris soin de dire, après avoir mentionné les a fonctionnaires et ouvriers visés par la loi du service civil », que ceux-ci restaient subordonnés à cette dernière loi, et il leur refusait à l'article 4, dernier paragraphe, le droit à l'arbitrage privé (i.e. en vertu d'une convention collective) et à celui prévu par la loi des Différends ouvriers de Québec, réservant le rôle d'arbitre, dans leur cas, à la Commission du Service civil.

Il ne faisait donc, à notre avis, aucun doute que la loi du Service civil continuait, seule à régir effectivement les employés gouvernementaux et que leur inclusion dans la loi des Différends entre les Services publics et leurs salariés n'avait été faite que pour leur enlever d'une manière explicite le droit de grève, limiter leur droit à l'association (article 6, 3e), et les astreindre aux sanctions prévues par cette loi.

Or, l'esprit et les dispositions de l'ancienne loi du Service civil nous obligent à conclure que toute forme valable de négociation et de revendication collectives leur était interdite par notre législation, la Commission du Service civil et le Lieutenant-gouverneur en conseil décidant de tout unilatéralement aux termes de cette loi. Les fonctionnaires avaient donc le droit à l'association mais ils ne pouvaient s'affilier à une centrale syndicale telles la C.S.N. ou le C.T.C., ni à aucune autre organisation syndicale groupant des salariés autres que des fonctionnaires.

Ils avaient le droit à l'accréditation syndicale, celui de négocier collectivement avec leur employeur, mais en pratique, cette convention collective ne pouvait exister que dans la mesure où l'Etat consentait à se lier et pour les matières quil voulait bien accepter de discuter avec les associations reconnues; autant dire rien en pratique.

LA GENÈSE IMMÉDLATE DE LA LOI

En février 1964 un comité parlementaire spécial était formé en vue d'étudier l'ensemble de la question des relations de travail de la fonction 
publique. Des rapports ont été soumis au gouvernement par ce Comité au printemps 1964, recommandant certains éléments de solution à cette question des relations du travail dans la fonction publique proprement dite ainsi que dans trois autres secteurs d'employés publics: le secteur hospitalier, le secteur scolaire et le secteur municipal. Des séances d'audition ont alors eu lieu, pour permettre aux associations patronales et syndicales intéressées de faire valoir leurs points de vue à ce sujet. Durant les trois jours qu'ont duré les séances, quatorze associations se sont fait entendre.

Pour ne considérer que le cas des "fonctionnaires " proprement dits, c'est-à-dire, les employés du gouvernement du Québec visés par la loi du Service civil (de la Fonction publique) le gouvernement, après avoir accepté dans son ensemble le rapport du Comité parlementaire, a exclu les fonctionnaires (sauf ceux qui sont au service de la Régie des alcools du Québec) de la définition du terme "salarié », les excluant par le fait même de l'application du Code du Travail. Le législateur, dans leur cas, devait élaborer une nouvelle loi de la Fonction publique, dans laquelle serait consacré le nouveau statut des fonctionnaires, et qui délimiterait du même coup l'ensemble de leurs relations collectives avec l'Etat.

C’est ce qui apparaît dans la première édition du Code du Travail, tel que sanctionné le 31 juillet 1964, à l'article $\mathrm{I}$, " $\mathrm{m}$ », 3a où l'on exclut les fonctionnaires régis par la loi du service civil de la définition de " salarié » pour les fins de ce Code.

De plus, à l'article 145 du Code de 1964, le législateur refusait aux fonctionnaires non membres d'une profession libérale (cf. art. 20 C.T., 1964) le droit de faire partie d'une association de salariés au sens du Code, et toute affiliation à une telle association.

Faisant suite à la décision des pouvoirs publics d'élaborer une loi refondue de la Fonction publique, on s'attacha d'abord à déterminer les groupes d'employés qui seraient éventuellement couverts par cette loi; puis, en novembre 1964 un scrutin de représentation syndicale fut tenu afin d'établir quel organisme, du Syndicat des fonctionnaires du Québec ou du Conseil général des employés du gouvernement de la province de Québec, serait habilité à représenter l'ensemble des fonctionnaires dans les négociations qui s'établiraient éventuellement avec le gouvernement. Le Syndicat des fonctionnaires provinciaux du Québec triompha, comme on le sait, lors de ce scrutin.

Le 3 février 1965, un comité conjoint d'étude était créé, formé de représentants gouvernementaux et syndicaux, lequel, pendant trois mois, a tenté d'élaborer un projet devant servir à la création d'une nouvelle loi de la Fonction publique. Les parties ne purent s'entendre et des rapports distincts furent présentés aux autorités gouvernementales. 
Finalement, le Bill 55, après modifications apportées à la toute dernière minute de la dernière session provinciale, sous la pression des organismes syndicaux, et dont on se souvient encore, est devenu la Loi actuelle de la Fonction publique, par sa sanction, en date du 6 août 1965.

Brève ANALYSE dE LA LOI

Quelques remarques s'imposent au sujet de cette loi. En premier lieu, elle replace les fonctionnaires sous la coupe du Code du Travail, en ce sens qu'ils ne sont plus exclus purement et simplement de sa définition du terme «salarié ». Le Code du Travail a été en conséquence amendé dans sa deuxième édition, celle de 1965. Celui-ci ménage certaines exceptions, toutefois, en stipulant à l'article $\mathrm{I}$, " $\mathrm{m}$ ", 3e, que n'est pas un "salarié " au sens du Code, " un fonctionnaire du gouvernement dont l'emploi est, au jugement de la Commission des Relations du Travail, d'un caractère confidentiel, tel que celui d'un conciliateur du ministère du travail, d'un inspecteur de la Commission, d'un employé du Conseil exécutif, du Conseil de la trésorerie, de la Commission de la fonction publique, du cabinet d'un ministre ou d'un directeur de personnel ». Le caractère de la " confidentialité » est ici soulevé, car l'énumération donnée dans le Code n'est pas limitative et peut prêter à une application très large selon les critères utilisés.

En principe, donc, la loi de la Fonction publique replace les fonctionnaires dans l'économie générale du Code du Travail et prend figure de texte dérogatoire au Code en apportant certaines modifications, certaines restrictions, aux prescriptions, aux droits et obligations prévus au Code pour l'ensemble des salariés, en ce qui a trait aux fonctionnaires en particulier.

Ceci peut signifier beaucoup pour les fonctionnaires, car l'économie générale du Code du Travail ainsi que ses critères d'interprétation et d'application, pourront à notre avis s'y appliquer chaque fois qu'une disposition stricte de la loi de la Fonction publique, ne viendra pas y apporter une dérogation précise.

Le droit d'association est donc acquis, au sens du Code du Travail. Cependant, ici encore, certaines particularités sont à noter. La reconnaissance est donnée dans la nouvelle loi de la Fonction publique, au syndicat des fonctionnaires provinciaux du Québec, nommément désigné dans son texte; mais à l'avenir, c'est à la Commission des relations du travail qu'il incombera de statuer en matière d'accréditation. C'est elle, de plus, qui doit statuer sur tout conflit relatif à l'inclusion ou à l'exclusion de tout employé ou des catégories d'employés dans un groupe donné (art. 70). Ceci, pour les fonctionnaires salariés et les ouvriers salariés; quant aux salariés enseignants, aux salariés membres des professions libérales, aux gradués d'université non organisés en profession fermée, ainsi qu'aux agents de la paix, ce n'est plus le Syndicat des 
fonctionnaires qui peut les représenter, mais des associations de chacun de ces groupes, lesquelles recevront une accréditation du lieutenantgouverneur en conseil, initialement, mais dont la C.R.T. se chargera par la suite, tout comme c'est le cas pour les fonctionnaires et ouvriers.

Certaines possibilités de regroupement des groupes professionnels entre eux, (i.e. les membres des professions libérales mentionnées au Code du Travail) et de ces derniers avec les gradués d'univrsité, à la condition d'une volonté exprimée à la majorité absolue des individus concernés, sont permises à l'article 72 , pour fin d'accréditation commune à une association formée de ces différents groupes.

Remarquons qu'il s'agit ici de quelque chose de différent de ce qui est déjà prévu à l'article 20 du Code du Travail pour les professionnels salariés dans les entreprises privées ou dans les autres services publics. En effet, les membres des professions mentionnées au Code du Travail doivent constituer, selon cet article 20 C.T. des groupes distincts pour fin d'accréditation et de négociation, alors que dans la Fonction publique, des groupes interprofessionnels peuvent exister.

Il y a donc danger, lorsqu'il s'agit d'apprécier le caractère de * salarié » d'un fonctionnaire, de transposer les critères de la C.R.T. développés à partir des services privés, et de les appliquer tels quels aux employés civils. Où doit-on s'arrêter dans la structure des fonctions gouvernementales? Donc, il y a un problème, ici, quant à la détermination des unités de négociation dans la fonction publique, à laquelle, à notre avis, on ne peut appliquer d'emblée les critères de détermination utilisés dans l'entreprise privée.

Quant au droit d'affiliation, il est reconnu dans la loi, à l'article 73, sauf pour les agents de la paix, gardiens de prison, gardes-chasse, inspecteurs des transports ou des autoroutes et autres préposés à des fonctions d'agent de la paix (art. 74). Une restriction est à souligner, quant au droit d'affiliation des fonctionnaires. L'affiliation n'est permise qu'à la condition que la constitution de l'association lui interdise de faire de la politique partisane, ou de participer au financement d'un parti poltiique. A cette condition, une association de fonctionnaires peut s'affilier à qui elle veut, du moment que la centrale syndicale en question respecte ces interdictions dans son contrat d'affiliation.

Quant au droit de grève, sauf pour les agents de la paix, il est acquis aux fonctionnaires, à condition qu'on se soit entendu préalablement sur la détermination des services essentiels et sur la façon de les maintenir, ou qu'à défaut d'entente entre les parties, la C.R.T. ait décidé à ce sujet. Il s'agit ici d'une disposition toute nouvelle dans nos lois du travail qui vraisemblablement prêtera flanc à toutes sortes de difficultés, étant donné le caractère ambigu du terme "essentiel ", des interprétations diverses auxquelles il peut donner lieu selon les cas, et 
de l'absence de tradition utile pour déterminer en pratique dans un secteur comme celui de la fonction publique.

Pour ce qui est de la négociation collective elle est en principe limitée aux salaires (art. 27 et suiv.), aux heures de travail, et aux congés (art. 52). Il s'agit ici d'un départ considérable de l'économie générale de la convention collective telle qu'envisagée par le Code du Travail.

Plusieurs limitations sont apportées à la négociation collective des fonctionnaires par le législateur:

a) la classification de la fonction publique est du ressort exclusif de la Commission de la fonction publique (art. 21, 22, 23, etc.) et il en est de même quant à la direction du travail d'un employé et à la définition de ses devoirs, où aucune restriction n'est apportée à ce sujet aux chefs ou aux sous-chefs dans un ministère donné (art. 25).

b) en matière de nominations et de promotions, la liste d'éligibilité est nécessaire et cette liste est fournie par la Commission de la Fonction publique elle-même. Un candidat à un poste doit être « éligible » en vertu de cette liste, sur laquelle il n'est inscrit qu'après un examen préalable (art. 38). Il est dit, de plus, à l'article 34 que la nomination ou la promotion d'un candidat ne peut dépendre de l'ancienneté dans le cas des fonctionnaires. L'ancienneté peut cependant être un critère de nomination ou de promotion pour les ouvriers, en cas de compétence égale.

c) en matière de congés statutaires (fêtes chômées) la loi ne semble pas permettre de négociation à ce sujet, car il y est dit que seuls les dimanches et les jours de fête fixés par la loi sont jours fériés à observer dans la fonction publique (art. 53).

d) pour ce qui touche à la discipline et aux congédiements, la loi ne semble pas donner prise à des procédures de contestation établies par convention collective. La loi est muette quant aux griefs et à la procédure de griefs. Alors qu'elle semble investir l'employeur et ses agents d'une autorité sans réplique relativement à l'exercice des pouvoirs disciplinaires. Ainsi quant aux absences non motivées du service, de même qu'en ce qui a trait aux suspensions pour inconduite ou négligence, aucun recours n'est prévu, semble-t-il, par la loi, au profit de l'employé ainsi pénalisé ou suspendu. Si un fonctionnaire est destitué, c'est la Commission elle-même qui fait enquête ot recommande la destitution. Ici, l'employé en cause a droit de se faire entendre pendant l'enquête, avec ses témoins, et à sa demande son dossier doit être transmis au Lieutenant-gouverneur en conseil avant que la révocation ou la destitution ne soit décrétée. Mais tout cela est toujours le fait de l'employé seul, non du syndicat, et 
toujours en vertu de la loi (art. 61) et non de procédures de griefs établies par voie de négociation.

La seule procédure de grief dont la loi fait mention explicitement comme pouvant être établie par voie de convention collective, c'est celle relative à la réclamation d'un employé qui se croirait lésé par une décision de la Commission quant à son classement à l'intérieur de la classification établie unilatéralement par la Commission (art. 24).

\section{BRÈVE APPRECLATION}

Cette nouvelle loi de la Fonction publique représente en dépit de ses lacunes évidentes, un progrès considérable dans le statut du fonctionnaire au Québec. N'y aurait-il pas lieu, toutefois, de scruter plus attentivement les restrictions dont est l'objet la convention collective dans la Fonction publique, quant à son contenu possible, et aux recours négociables en matière de griefs, par exemple? De plus, en ce qui touche aux ouvriers, il importe de souligner que contrairement à la demande des syndicats ils ne sont pas régis uniquement par le Code du Travail, mais ils sont assimilés entièrement, ou presque aux fonctionnaires, sauf quant à l'utilisation du critère d'ancienneté en matière de promotion. Les employés de l'Etat ont donc la substance des droits consacrés par le droit du travail mais ils n'en ont pas l'extension dans leur application. Il serait prématuré, à ce stage de porter un jugement de valeur définitif et global sur le bien-fondé ou le mal-fondé de ces restrictions apportées par le législateur à la liberté contractuelle dans les relations de travail au niveau de la Fonction publique. Le temps se chargera sans doute, et assez tôt, à notre avis, d'en faire ressortir les éléments positifs aussi bien que ceux qui seraient de nature à faire obstacle à l'établissement de relations collectives valables dans un secteur aussi vital de notre économie.

Peut-on penser, toutefois, que les employés gouvernementaux, étant en principe régis par le Code du Travail, et que la loi de la Fonction publique n'apparaissant que comme une "dérogation " partielle aux dispositions de ce Code, la convention collective, dans les faits, prendra une importance plus grande, étendra son empire aux dépens des vieilles conceptions du droit administratif? Nous pouvons le prévoir surtout si on se rappelle la déclaration du premier ministre, lors du débat sur le Bill 55 en chambre, où il affirmait que la loi des parties primerait toujours sur les règlements que pourrait passer par la suite la Commission de la fonction publique. Ce qui laisserait supposer qu'aucun règlement de cette Commission ne pourrait venir en conflit avec les ententes préalablement faites entre les syndicats et les pouvoirs publics. 